$16^{\circ}$ USIHC - Congresso Internacional de Ergonomia e Usabilidade de

\title{
OBJETO DE APRENDIZAGEM EDUMOBILE - ESTRATÉGIAS PEDAGÓGICAS PARA O USO DA M-LEARNING EM SALA DE AULA: DO PLANEJAMENTO À IMPLEMENTAÇÃO
}

\section{EDUMOBILE LEARNING OBJECT - PEDAGOGICAL ESTRATEGIES FOR THE USE OF M-LEARNING IN A CLASSROOM: FROM PLANNING TO IMPLEMENTATION}

\author{
Anna Helena Silveira Sonego ${ }^{1}$, \\ Ana Carolina Ribeiro Ribeiro², \\ Leticia Rocha Machado ${ }^{3}$, \\ Patricia Alejandra Behar ${ }^{4}$, \\ Universidade Federal do Rio Grande do Sul \\ (1)sonego.anna@gmail.com \\ (2) carolribeiro2@gmail.com \\ (3) leticiarmachado@gmail.com \\ (4)pbehar@terra.com.br
}

M-Learning, Dispositivos Móveis, Estratégias Pedagógicas.

Este artigo versa sobre o planejamento, desenvolvimento e implementação do objeto de aprendizagem EduMobile Estratégias pedagógicas para o uso da M-Learning em sala de aula. A metodologia utilizada foi a ConstruMED. O objeto apresenta uma navegabilidade não linear, abordando elementos da usabilidade e pedagógicos que possibilitem uma reflexão sobre a M-Learning.

\section{M-Learning, Mobile Devices, Pedagogical Strategies}

This article deals with the planning, development and implementation of the learning object EduMobilePedagogical strategies for the use of M-Learning in the classroom. The methodology was ConstruMED. The object presents a non-linear navigability, approaching usability and pedagogical elements that allow a reflection on $M$ Learning. 


\section{$16^{\circ}$ \\ ERGODESIGN USIHC CINAHPA}

\section{Introdução}

O objetivo desse artigo é apresentar o processo de planejamento, desenvolvimento e implementação do Objeto de Aprendizagem (OA) EduMobile Estratégias pedagógicas para o uso da MLearning em sala de aula. Esse objeto tem como objetivo auxiliar o professor na construção de estratégias pedagógicas que possibilitem a formação do sujeito, no âmbito educacional, para utilizar a tecnologia móvel.

A cada ano aumenta o número de dispositivos móveis no mundo. $\mathrm{Na}$ "Pesquisa Brasileira de Mídia" (BRASIL, 2014) foi destacado que o computador de mesa é o equipamento mais utilizado para acessar a internet. O celular aparece na segunda posição $(66 \%)$ e na terceira o tablet (7\%). Estes dados mostram que o Brasil está utilizando o smartphone para diferentes finalidades, incluindo o acesso às ferramentas digitais da internet. Este interesse ocorre por diferentes motivos, entre eles a facilidade na aprendizagem do uso dos aparelhos (principalmente pela interação na tela touch screen), a mobilidade e a rapidez na comunicação.

Este novo panorama no uso destas tecnologias digitais só é possível devido às condições de mobilidade, flexibilidade e conectividade que estes aparelhos oferecem aos usuários, já que permite a utilização qualquer lugar e horário (MOURA, 2011). Portanto, acredita-se na viabilidade de sua utilização para o desenvolvimento da M-Learning, ou seja, aprendizagem móvel, já que permite a ampliação das formas de comunicação e de compartilhamento de informações e do conhecimento.

Com a integração das tecnologias digitais no meio escolar, principalmente as móveis, surge também a necessidade de recorrer a conteúdos e materiais que venham servir de subsídios para aprimorar a prática docente. Um Objeto de Aprendizagem (OA) poderá ser capaz de apresentar esses elementos como exemplos a serem seguidos ou, ainda, proporcionar condições para construção de novas atividades e estratégias pedagógicas a partir do próprio objeto. Um OA pode ser definido como $16^{\circ}$ Ergodesign - Congresso Internacional de Ergonomia e Usabilidade de Interfaces Humano Tecnológica: Produto, Informações Ambientes Construídos e Transporte

$16^{\circ}$ USIHC - Congresso Internacional de Ergonomia e Usabilidade de Interfaces Humano Computador

CINAHPA | 2017 - Congresso Internacional de Ambientes Hipermídia para Aprendizagem.

qualquer material digital (vídeo, site, etc.) que possui finalidade educativa (BEHAR, 2009). Nesse sentido, torna-se relevante, para a M-Learning, que os materiais sejam interativos e responsivos, podendo ser manipulados em qualquer dispositivo móvel. Portanto, o desenvolvimento de um objeto de aprendizagem poderá auxiliar professores nas suas práticas pedagógicas para a M-Learning, já que os conteúdos abordados poderão, além de ser acessados nos dispositivos móveis, apresentar reflexões pertinentes sobre o tema. Desse modo, o presente artigo apresenta o planejamento, desenvolvimento e a implementação de um objeto de aprendizagem voltado para a M-Learning.

No intuito de contemplar as temáticas que serão tratadas no decorrer do artigo, a organização do trabalho foi realizada em quatro seções. $\mathrm{Na}$ segunda seção são abordados os conceitos sobre a aprendizagem móvel, além das possibilidades de utilização dos dispositivos móveis. Já na terceira são descritos os procedimentos metodológicos do estudo. Na quarta seção são elencados os resultados, obtidos a partir da construção do OA EduMobile. E, por fim, na quinta seção são apresentadas as considerações, não-finais, que indicam possibilidades de uso do OA pelos professores.

\section{0 uso dos dispositivos móveis para o desenvolvimento da M-Learning}

A M-Learning é um tipo de aprendizagem que se desenvolve com o uso dos dispositivos móveis separados ou em combinação com outras tecnologias da informação e da comunicação (UNESCO, 2013). Sendo assim, consideram-se como dispositivos móveis os smartphones e tablets, entre outros aparelhos, que possuam conexão com a internet sem fio, podendo ser wireless, $3 \mathrm{G}$ ou $4 \mathrm{G}$. O que diferencia estes equipamentos de outros é a capacidade que oferecem quanto à mobilidade, flexibilidade $\mathrm{e}$ tamanho, que facilita o seu deslocamento para qualquer lugar. Portanto, pode-se fazer uso para fins educacionais, ultrapassando o uso apenas de entretenimento, mas utilizando-os em momentos que oportunize aos estudantes construir e intensificar os conhecimentos (LEITE, 2014).
Realização:

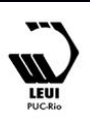




\section{$16^{\circ}$ \\ ERGODESIGN USIHC CINAHPA}

Desse modo, a mediação de atividades com os dispositivos móveis proporcionam condições aos docentes para inovação em todas as áreas, possibilitando, nas suas práticas pedagógicas, perpassar por movimentos que permitam experimentar, utilizar e explorar as Tecnologias de Comunicação e Informação (TIC) dentro e fora do âmbito escolar. Sendo assim, considera-se que o desenvolvimento da M-Learning a partir do uso dos dispositivos, pode gerar situações que permitam criar novas possibilidades e desafios no processo de ensino e de aprendizagem.

Para tanto, não se pode afirmar que o simples fato de utilizar um dispositivo móvel, como um smartphone ou tablet, para desempenhar uma atividade em aula, seja caracterizado como aprendizagem móvel. O docente deve ter um planejamento que envolva um conteúdo curricular, um material didático e uma estratégia pedagógica definindo o modo de utilização de dispositivos móveis. Estratégias pedagógicas podem ser consideradas como um conjunto de ações educacionais colocadas em prática pelo professor, no qual visam alcançar os objetivos que levam à construção do conhecimento (BEHAR, 2013).

Neste contexto, considerando as características citadas para a M-Learning, será possível utilizar os dispositivos móveis em sala de aula com o objetivo de enriquecer o processo de ensino e aprendizagem, aplicando estratégias pedagógicas inovadoras.

Portanto, na próxima seção serão tratados o conceito e as características dos objetos de aprendizagem a fim de compreender as possíveis utilizações para a M-Learning.

\section{Objeto de Aprendizagem}

Como citado anteriormente, no contexto educacional, vê-se uma crescente inclusão das tecnologias de informação e comunicação na sala de aula. Dessa forma, a produção de Materiais Educacionais Digitais (MED) tem crescido, principalmente, nos meios acadêmicos, como uma possibilidade para a apresentação de conteúdos e materiais de uma forma mais interativa. Muitos $16^{\circ}$ Ergodesign - Congresso Internacional de Ergonomia e Usabilidade de Interfaces Humano Tecnológica: Produto, Informações Ambientes Construídos e Transporte

$16^{\circ}$ USIHC - Congresso Internacional de Ergonomia e Usabilidade de Interfaces Humano Computador

CINAHPA | 2017 - Congresso Internacional de Ambientes Hipermídia para Aprendizagem.

desses materiais são disponibilizados em forma de objetos de aprendizagem, como uma unidade de ensino reutilizável, configurando-se como um recurso que pode enriquecer o espaço pedagógico.

De acordo com Behar et al (2009, p.67), entendese por objeto de aprendizagem "qualquer material digital, como, por exemplo, textos, animações, vídeos, imagens, aplicações, páginas web de forma isolada ou em combinação, com fins educacionais". Dessa forma, um OA pode ser material que possua fins educativos e embasamento pedagógico. Além disso, são materiais que possam ser destinados a situações de aprendizagem tanto na modalidade à distância, quanto presencial. Assim, neste estudo, compreende-se objeto de aprendizagem como qualquer material/recurso digital que seja formado por diferentes mídias, estratégias e desafios que propiciem ao usuário um espaço de aprendizagem. Esses materiais podem ser utilizados como módulos de um determinado conteúdo ou como uma estrutura completa.

Torrezzan e Behar (2013) destacam que a principal característica de um objeto consiste na possibilidade de ser reutilizável, abrangendo diferentes conteúdos, estratégias pedagógicas, por parte do professor, e estilos de aprendizagem, por parte do aluno. Por não apresentar uma estrutura linear, na sua maioria, permite ao usuário a navegação de acordo com seu interesse e percepção sobre o tema, configurando-se, assim, como uma importante ferramenta educacional.

Entende-se que a utilização de OAs na prática pedagógica viabiliza aos sujeitos uma participação mais ativa em seu processo de aprendizagem, pois o material oferece diferentes meios pelos quais o aluno poderá construir seu conhecimento. Além disso, por ser disponibilizado em diversas mídias, pode subsidiar os mais diferentes tipos de prática pedagógica, proporcionando espaços de interação e interatividade aos seus usuários. Dessa forma, na produção de um OA é importante que o professor possua um conhecimento profundo de sua área de atuação, além das diferentes formas de aprendizagem de seus alunos, para que o objeto seja de auxílio a sua prática e consistente com os 


\section{$16^{\circ}$ \\ ERGODESIGN USIHC CINAHPA}

$16^{\circ}$ Ergodesign - Congresso Internacional de Ergonomia e Usabilidade de Interfaces Humano Tecnológica: Produto, Informações Ambientes Construídos e Transporte

$16^{\circ}$ USIHC - Congresso Internacional de Ergonomia e Usabilidade de Interfaces Humano Computador

CINAHPA | 2017 - Congresso Internacional de Ambientes Hipermídia para Aprendizagem. seus objetivos. Desta forma, o OA poderá auxiliar os professores no desenvolvimento de práticas pedagógicas para a M-Learning, já que suas possibilidades de aplicação são inúmeras e enriquecedoras.

A seguir, é explicitada a metodologia utilizada para a construção do objeto de aprendizagem EduMobile.

\section{Metodologia}

Para o desenvolvimento do OA EduMobileEstratégias pedagógicas para o uso da $M$ Learning em sala de aula contou-se com a atuação de uma equipe interdisciplinar composta por educadores, programadores e webdesigners. A metodologia utilizada foi a ConstruMED, que consiste em construir materiais educacionais digitais baseados no design pedagógico (TORREZZAN, 2014). De acordo com Torrezzan e Behar (2013), o Design Pedagógico é uma concepção teórica que visa "orientar a aplicação de recursos digitais em materiais educacionais por meio do concomitante planejamento técnico, gráfico e pedagógico". Cabe destacar que nessa pesquisa entende-se objeto de aprendizagem como sinônimo de material educacional digital.

A construção do objeto EduMobile seguiu as cinco etapas propostas pela metodologia ConstruMED:

ETAPA 1 - Preparação: etapa de definição da equipe e das características básicas do tema/usuários. Abrange a organização da equipe de trabalho, a definição do tema abordado pelo MED/OA, a caracterização do público-alvo e a elaboração dos objetivos pedagógicos.

ETAPA 2 - Planejamento: etapa de elaboração do conteúdo e delimitação do mesmo; o planejamento das atividades envolvendo a aplicação prática da teoria estudada; o levantamento de materiais de apoio; a preparação do roteiro do OA abrangendo título do objeto, objetivo e resumo geral do conteúdo; a definição da estrutura, a partir do conteúdo de cada página e das diferentes mídias que serão incluídas; a definição e a elaboração dos recursos pedagógicos e tecnológicos; o planejamento da identidade visual das interfaces gráficas; o levantamento de critérios de usabilidade e acessibilidade; a elaboração do mapa de navegação do OA; e, por fim, a seleção de softwares e códigos de programação informática passíveis de serem utilizados para implementar o OA.

ETAPA 3 - Implementação: etapa de construção digital do objeto de aprendizagem, que abrange o desenvolvimento propriamente dito, incluindo a elaboração dos primeiros protótipos dos objetos até alcançar a sua versão final (após a etapa 4 avaliação). Implica a análise e revisão do conteúdo elaborado e disposto em cada módulo e interface do OA; a organização do texto de apresentação disponibilizado na página inicial e das suas páginas auxiliares: Material de Apoio, Glossário, Créditos etc; idealização do wireframe das interfaces gráficas, ou seja, setorização, em cada interface, dos elementos que ela contém (logo, textos, botões de navegação, imagens etc.); implementação do OA a partir do roteiro, conteúdo pedagógico, mapa de navegação e wireframe.

ETAPA 4 - Avaliação: etapa de análise do funcionamento do OA e da adequação aos objetivos técnicos, gráficos e pedagógicos. A partir dessa análise são realizados ajustes finais que incluem a testagem da utilização do MED/OA a partir da visão do usuário/aluno, anotações sobre a necessidade de reparos e aprimoramentos; testes de funcionamento de links, botões e animações; análise dos materiais de acordo com os objetivos pedagógicos e do design gráfico das interfaces; utilização do OA em diferentes navegadores.

ETAPA 5 - Distribuição: etapa de armazenamento e disponibilização do MED/OA, destinada à distribuição do OA construído e já avaliado. Para a disponibilização em sites e repositórios são descritos os metadados que serão solicitados durante o processo de cadastro e que são destinados a caracterizar o referido OA para este ser encontrado em buscas online.

Realização:




\section{$16^{\circ}$ \\ ERGODESIGN USIHC CINAHPA}

\section{Resultados}

O objeto de aprendizagem EduMobile (Figura 1) foi desenvolvido com o objetivo de auxiliar na construção de estratégias pedagógicas que propiciem ao professor utilizar dispositivos móveis de forma construtiva, ou seja, buscando a construção do conhecimento.

Cabe salientar que o objeto EduMobile faz parte de um trabalho em nível de Doutorado na área da Educação na Universidade Federal do Rio Grande do Sul, desenvolvido em paralelo com pesquisas realizadas no Núcleo de Tecnologia Digital Aplicada à Educação (NUTED) na mesma Universidade.

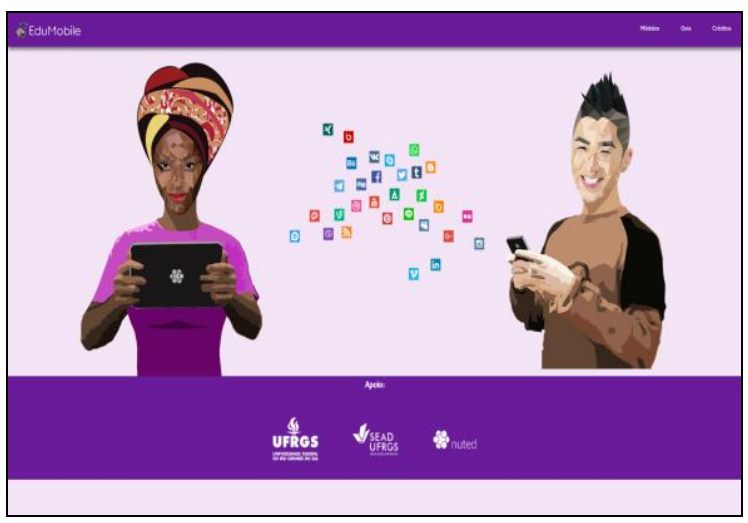

Figura 1. Tela inicial do OA EduMobile.

As etapas de desenvolvimento do referido objeto, conforme citado anteriormente, utilizaram como apoio a metodologia ConstruMED. Sendo assim, estas serão detalhadas a seguir.

\section{ETAPA 1 - Preparação}

Para atender a esta etapa, inicialmente, foram realizadas reuniões semanais com a equipe interdisciplinar composta por pedagogos e designers. O público-alvo do objeto foi definido nesta etapa, sendo professores e tutores que desejam trabalhar com a M-Leanring, tanto presencialmente como virtualmente.

\section{ETAPA 2 - Planejamento}

O objeto foi pensado e planejado com um layout intuitivo, onde a metáfora utilizada contemplasse $16^{\circ}$ Ergodesign - Congresso Internacional de Ergonomia e Usabilidade de Interfaces Humano Tecnológica: Produto, Informações Ambientes Construídos e Transporte

$16^{\circ}$ USIHC - Congresso Internacional de Ergonomia e Usabilidade de Interfaces Humano Computador

CINAHPA | 2017 - Congresso Internacional de Ambientes Hipermídia para Aprendizagem.

as diferenças entre alunos e professores no uso das tecnologias.

Foi desenvolvido o Storyboard do objeto (Figura 2) no intuito de auxiliar a construção da navegação que seria adotada no OA.

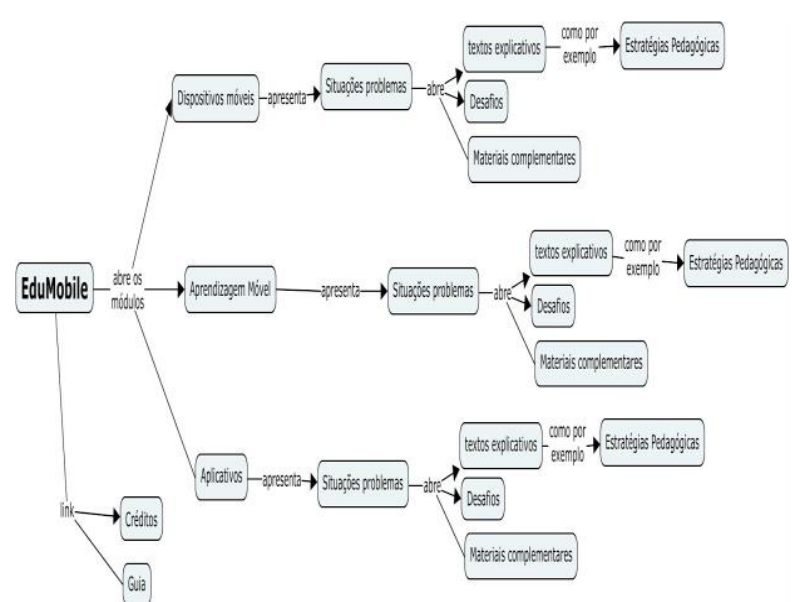

Figura 2. Storyboard do OA EduMobile.

\section{ETAPA 3 - Implementação}

No que se refere ao conteúdo, para atender ao objetivo do objeto, foram desenvolvidos quatro módulos que abrangem os seguintes temas:

- Dispositivos móveis, que aborda diferentes perspectivas e conceitos sobre o tema, além de suas principais características, tipos de dispositivos e as possibilidades de uso educacional. Os materiais de apoio referentes a esse módulo buscam auxiliar o usuário a compreender o contexto de uso dos dispositivos móveis no país, os principais sistemas operacionais utilizados e o conceito na educação;

- Aplicativos, que apresenta a definição de aplicativos (apps), principalmente voltados aos dispositivos móveis, suas principais funções, categorias e seu uso na educação. Para isso, são abordadas as formas de utilização dos aplicativos no cotidiano no intuito de refletir sobre as possibilidades de uso no contexto escolar. Também são tratadas formas de construção de aplicativos, visando atender objetivos pedagógicos específicos e a autoria dos alunos;
Realização:
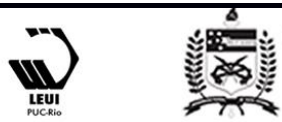


\section{$16^{\circ}$ \\ ERGODESIGN USIHC CINAHPA}

- M-Learning, que trata da definição de aprendizagem móvel e seus principais desafios, atentando a aspectos econômicos e de acessibilidade, por exemplo. Além disso, são abordadas as possibilidades e tendências desse tipo de aprendizagem, como a criação de conteúdo por alunos e as facilidades de acesso a diversos tipos de materiais em qualquer momento e espaço. Os Materiais de Apoio trazem a comparação entre a aprendizagem móvel e outros tipos de aprendizagem, além das práticas pedagógicas relacionadas a M-Learning;

- Estratégias pedagógicas, que aborda as diferentes formas de utilização de dispositivos móveis e aplicativos na educação, além de estratégias que podem ser adotadas pelo professor de modo a promover a aprendizagem móvel.

Todos os módulos apresentam Materiais de Apoio como pode ser visto na Figura 3, que são disponibilizados através de diferentes mídias e buscam propiciar uma abordagem abrangente dos assuntos.

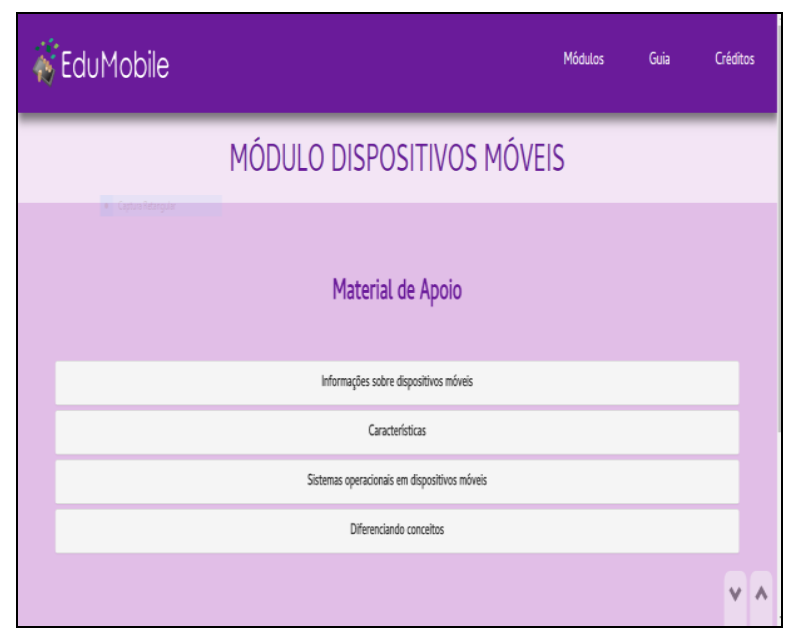

Figura 3. Tela do Material de Apoio do Módulo de Dispositivos Móveis.

São apresentados também Desafios, conforme as Figuras 4 e 6, a fim de problematizar os assuntos abordados e promover ao usuário espaços de reflexão e interação. $16^{\circ}$ Ergodesign - Congresso Internacional de Ergonomia e Usabilidade de Interfaces Humano Tecnológica: Produto, Informações Ambientes Construídos e Transporte

$16^{\circ}$ USIHC - Congresso Internacional de Ergonomia e Usabilidade de Interfaces Humano Computador

CINAHPA | 2017 - Congresso Internacional de Ambientes Hipermídia para Aprendizagem.

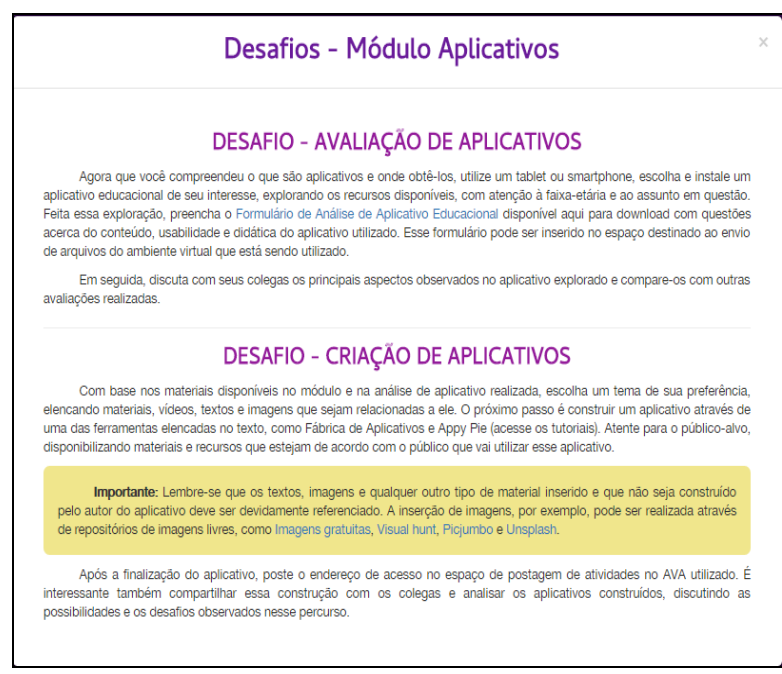

Figura 4. Tela dos Desafios do Módulo de Aplicativos.

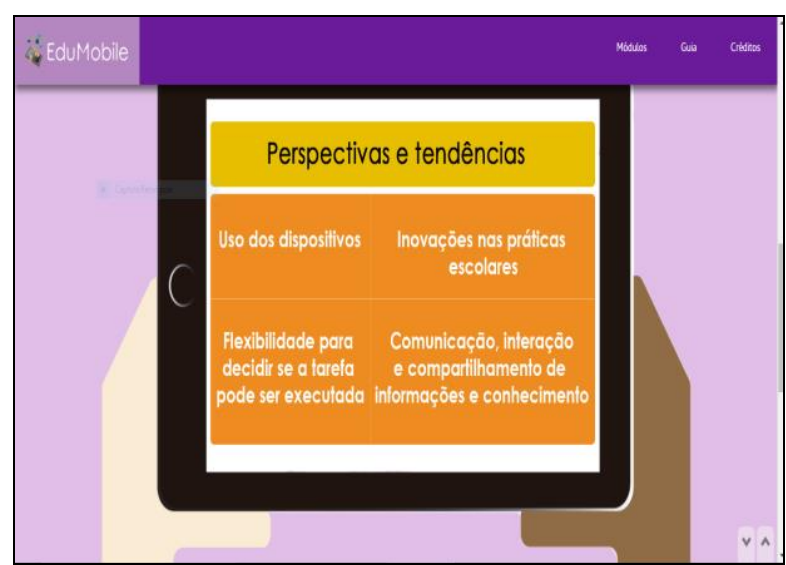

Figura 5. Tela do Módulo M-Learning.

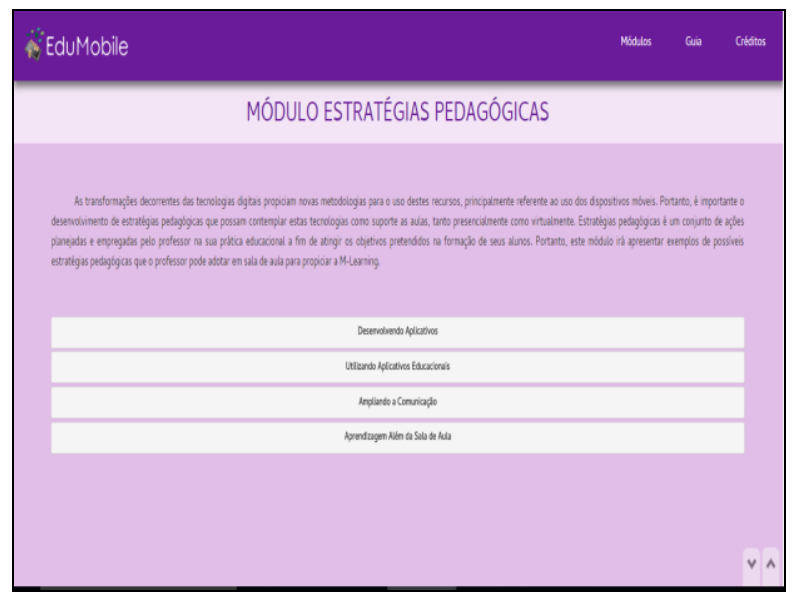

Figura 6. Tela dos Desafios do Módulo de Estratégias Pedagógicas. 


\section{$16^{\circ}$ \\ ERGODESIGN USIHC CINAHPA}

Por fim, há um Guia que apresenta as formas de uso do objeto e os pré-requisitos técnicos para o funcionamento do mesmo.

Os módulos do objeto de aprendizagem poderão ser acessados na ordem em que se apresentam na interface ou segundo a escolha do usuário, de modo a se respeitar o raciocínio de cada sujeito (Figura 7).

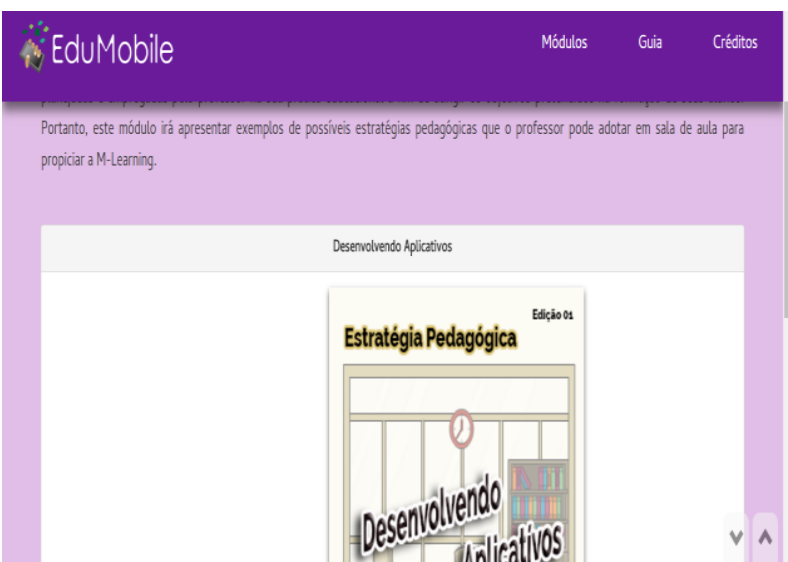

Figura 7. Tela de exemplo de multimídia do módulo de estratégias pedagógicas.

Todo o material multimídia incluído no OA foi acompanhado por recursos de acessibilidade, como legendas e resenhas no caso de vídeos. Também foram considerados os critérios de usabilidade para o desenvolvimento desses recursos. Para ser utilizado em dispositivos móveis o site foi construído de forma responsiva, possibilitando que o mesmo se adapte ao tamanho da tela do aparelho eletrônico que está utilizando.

As interfaces do OA EduMobile foram criadas objetivando ser amigáveis e de fácil utilização, não necessitando de conhecimentos aprofundados ou específicos sobre os recursos informáticos. As interfaces seguem a estrutura padrão de navegação utilizada pela maioria dos sites encontrados na Web. O EduMobile pode ser acessado através do endereço: http://www.nuted.ufrgs.br/oa/edumobile/

\section{ETAPA 4 - Avaliação}

O objeto de aprendizagem EduMobile- Estratégias pedagógicas para o uso do M-Learning em sala de aula foi utilizado em um curso de extensão $16^{\circ}$ Ergodesign - Congresso Internacional de Ergonomia e Usabilidade de Interfaces Humano Tecnológica: Produto, Informações Ambientes Construídos e Transporte

$16^{\circ}$ USIHC - Congresso Internacional de Ergonomia e Usabilidade de Interfaces Humano Computador

CINAHPA | 2017 - Congresso Internacional de Ambientes Hipermídia para Aprendizagem.

ofertado na UFRGS para professores de diferentes níveis da Educação Básica. O curso de extensão foi oferecido na modalidade semipresencial. No entanto, não foi possível realizar uma avaliação formal já que o cronograma do curso sofreu mudanças devido às ocupações nas salas de aula da Universidade onde o curso ocorreu. Desta forma, sua avaliação não pode ser finalizada. Assim, o objeto será utilizado em uma disciplina de graduação ofertada para os cursos de licenciatura da mesma Universidade, no qual serão avaliados os seguintes pontos de análise: 1) interface gráfica e interatividade homem-máquina (usabilidade, acessibilidade e se as estruturas técnicas e gráficas do OA apoiam os objetivos pedagógicos da proposta), conteúdo e metodologia adotada. Os dados serão coletados através de questionários e de observação participante. As necessidades que surgirem a partir dos dados analisados serão devidamente anotadas e repassadas para os ajustes. Após o aperfeiçoamento, o material poderá ser utilizado tanto online como em CD/DVD, possibilitando uma maior abrangência do material. Pretende-se que esse MED seja utilizado em qualquer curso de formação voltado para professores, tutores e alunos da Educação a Distância que busquem informações sobre este assunto.

\section{Considerações Finais}

A cada ano cresce o número de dispositivos móveis que possuem por objetivo a mobilidade e a conectividade. A educação precisa cada vez mais incorporar estas ferramentas em sala de aula, o que propicia uma discussão aprofundada sobre como potencializar o uso destes recursos nas práticas pedagógicas. Acredita-se que este uso possa contribuir significativamente para o ensino e a aprendizagem. Portanto, o objeto EduMobile foi desenvolvido no intuito de auxiliar professores de diferentes áreas e diferentes modalidades a refletir sobre possíveis estratégias pedagógicas a fim de contemplar as características da M-Learning.

Nesse sentido, o presente artigo apresentou o planejamento, desenvolvimento e implementação do objeto EduMobile. Para tanto, se fez necessário
Realização:

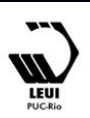




\section{$16^{\circ}$ \\ ERGODESIGN USIHC CINAHPA}

$16^{\circ}$ Ergodesign - Congresso Internacional de Ergonomia e Usabilidade de Interfaces Humano Tecnológica: Produto, Informações Ambientes Construídos e Transporte

$16^{\circ}$ USIHC - Congresso Internacional de Ergonomia e Usabilidade de Interfaces Humano Computador

CINAHPA | 2017 - Congresso Internacional de Ambientes Hipermídia para Aprendizagem. desenvolver estratégias pedagógicas que pudessem apoiar e incentivar estas etapas através da utilização dos dispositivos móveis. Nessa perspectiva, acredita-se que as estratégias pedagógicas são capazes de minimizar os desafios que os dispositivos podem apresentar para os professores, ampliando as possibilidades tanto para a ação docente quanto para os discentes em seus processos de aprendizagem. Ou seja, o professor além de poder utilizar as estratégias apresentadas no referido objeto de aprendizagem, obterá subsídios para desenvolver novas estratégias de acordo com seu contexto escolar.

O uso da metodologia ConstruMED auxiliou no processo de desenvolvimento do objeto, já que as suas etapas possibilitaram uma construção interdisciplinar contínua, a fim de contemplar todos os tópicos propostos no OA.

No contexto educacional, vê-se uma crescente necessidade de inclusão dos dispositivos móveis. Paralelamente, a produção de materiais educacionais digitais, como os objetos de aprendizagem, tem crescido, principalmente, nos meios acadêmicos, como uma possibilidade para a apresentação de conteúdos e materiais de uma forma mais interativa e lúdica. Dessa forma, entende-se que o desenvolvimento de materiais, a exemplo do EduMobile, pode contribuir de maneira significativa, tanto com a prática pedagógica, quanto com a difusão de tecnologias e ferramentas que contemplem diferentes áreas de formação no processo de ensino e aprendizagem.

\section{Referências}

BEHAR, P.; MACEDO, A. L.; SOUZA, A. P. F. C.; BERNARDI, M. Objetos de Aprendizagem para a Educação a Distância. In: Modelos pedagógicos em educação a distância. Porto Alegre: Artmed, 2009.

BRASIL, Presidência da República. Secretaria de Comunicação Social. Pesquisa brasileira de mídia: hábitos de consumo de mídia pela população brasileira. Brasília: Secom. 2014.
LEITE, B. S. (2014), "M-Learning: o uso de dispositivos móveis como ferramenta didática no Ensino de Química”. Revista Brasileira de Informática na Educação, Volume 22, N.3. http://www.academia.edu/11729289/M-

Learning_o_uso_de_Dispositivos\%C3\%B3veis_co mo_ferramenta_did\%C3\%A1tica_no_Ensino_de_ Qu\%C3\%Admica

MOURA, A. M. C. Apropriação do telemóvel como ferramenta de mediação em Mobile Learning: estudos de caso em contexto educativo. 2011. 630f. (tese de doutorado). (Universidade do Minho, Braga, PT, 2011). Disponível em: http://repositorium.sdum.uminho.pt/handle/1822/1 $\underline{3183 .}$.

TORREZZAN, Cristina. A. W.; BEHAR, P. A. . Competências para a Construção de Materiais Educacionais Digitais Baseados no Design Pedagógico. In: Patricia Alejandra Behar. (Org.). Competências em Educação a Distância. 1ed.Porto Alegre: Penso, 2014, v. 1, p. 237-262.

UNESCO. (2013), "Policy Guidelines for Mobile Learning”. Publicado pela Organização das Nações Unidas para a Educação, a Ciência e a Cultura (UNESCO), 7, place de Fontenoy, 75352 Paris 07 SP, France. A tradução para o português desta publicação foi produzida pela Representação da UNESCO no Brasil. Disponível em: http://www.unesco.org/new/pt/brasilia/about-thisoffice/single-

view/news/diretrizes de politicas da unesco para a aprendizagem movel pdf only/\#.VZ5E0vgju1 $\underline{E}$

BEHAR, P. Competências em Educação a Distância. Penso: Porto Alegre, 2013.

TORREZZAN, C. ConstruMed: Metodologia para a construção de materiais educacionais digitais baseados no design pedagógico. 2014. Tese (Doutorado em Informática na Educação), Programa Pós-graduação em Informática na Educação, Centro Interdisciplinar de Novas Tecnologias na Educação, Universidade Federal do
Realização:

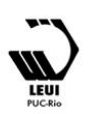




\section{$16^{\circ}$ \\ ERGODESIGN USIHC CINAHPA}

$16^{\circ}$ Ergodesign - Congresso Internacional de Ergonomia e Usabilidade de Interfaces Humano Tecnológica: Produto, Informações Ambientes Construídos e Transporte

$16^{\circ}$ USIHC - Congresso Internacional de Ergonomia e Usabilidade de Interfaces Humano Computador

CINAHPA | 2017 - Congresso Internacional de Ambientes Hipermídia para Aprendizagem.
Rio Grande do Sul, Porto Alegre, 2014. Disponível em:

< http://www.lume.ufrgs.br/handle/10183/106458>.

WILEY, D. Connecting learning objects to instructional design theory: A definition, a metaphor, and a taxonomy . 2000. In: WILEY, D (Ed.) Instructional Use of Learning Objects.

Association of Educational Communications and Technology.
Agradecimentos: A Secretaria de Educação à Distância da UFRGS, a equipe pedagógica e do design que desenvolveram o Objeto de Aprendizagem EduMobile. 